\title{
Dependência tabágica, assertividade e alexitimia em doentes cardíacos ${ }^{1}$
}

\author{
Vânia Rocha \\ Marina Prista Guerra \\ Maria Júlia Maciel \\ Universidade do Porto, Porto, Portugal
}

\begin{abstract}
Resumo: O tabagismo é uma das principais causas evitáveis de morte, aumentando acentuadamente o risco de infarto do miocárdio. Este último está associado a certas variáveis psicossociais relacionadas com o tabagismo, nomeadamente a alexitimia e a falta de assertividade. O objetivo do presente artigo foi caracterizar os dois tipos de dependência tabágica (fisiológica e comportamental), a assertividade e a alexitimia, e avaliar a relação das variáveis entre si, em 30 doentes homens internados com infarto. Foram aplicados o Teste de Fagerström, o Questionário de Glover-Nilsson, a Escala de "Rathus" e a TAS-20. Os resultados indicam que a alexitimia encontra-se relacionada com a dependência comportamental e com a assertividade, sugerindo que os sujeitos menos assertivos e com dificuldade em gerir afetos negativos e situações de estresse, podem recorrer ao tabagismo como mecanismo de enfrentamento. Nas intervenções voltadas à interrupção do hábito de fumar em doentes infartados deve-se enfatizar as competências sociais e de regulação emocional.
\end{abstract}

Palavras-chave: tabagismo, assertividade, alexitimia, infarto do miocárdio.

\section{Tobacco dependence, assertiveness and alexithymia in cardiac patients}

\begin{abstract}
Smoking is a major cause of preventable death, which greatly increases the risk of suffering a myocardial infarction. This cardiac event is associated with certain psychosocial variables which have been related to smoking, such as alexithymia and non assertiveness. The general purpose of this study is to evaluate both types of tobacco dependence (physiological and behavioural), assertiveness and alexithymia, as well as the relation established between them in 30 male patients hospitalized with myocardial infarction. The Fagerström Test, the Glover-Nilsson Questionnaire, the Rathus Assertiveness Schedule and TAS-20 were used. The results point out that alexithymia is related with behavioural dependence and assertiveness, which suggest that cigarette smoking is used by the subjects as a coping mechanism, due to their difficulties in expressing emotions assertively, regulating negative affects and dealing properly with stressful events. Social skills and emotional regulation should be emphasized in psychological interventions for smoking cessation with cardiac patients
\end{abstract}

Keywords: smoking, assertiveness, alexithymia, myocardial infarction.

\section{Tabaquismo, asertividad y alexitimia en pacientes cardíacos}

Resumen: Fumar es una causa importante de muertes evitables, aumentando el riesgo de infarto de miocardio. Esto se asocia con determinadas variables psicosociales relacionadas con el tabaquismo, como la alexitimia y la falta de asertividad. El objetivo consiste en la valoración de los dos tipos de dependencia al tabaco (fisiológica y comportamental), la asertividad y alexitimia, y evaluar la relación entre las variables en 30 pacientes masculinos hospitalizados con infarto. Fueron realizados el Test de Fagerström, el Cuestionario de Glover-Nilsson, la Escala de Asertividad de Rathus y la TAS-20. Los resultados indican que la alexitimia está relacionada con la dependencia comportamental y con la asertividad, lo que sugiere que la gente recurre al tabaquismo como mecanismo de enfrentamiento, debido a que no son capaces de expresar asertivamente sus emociones y de controlar sentimientos negativos y situaciones de estrés. Por eso, se debe incidir en que estos pacientes dejen de fumar, a nivel de competencias sociales y de regulación emocional.

Palabras clave: tabaquismo, asertividad, alexitimia, infarto de miocardio.

O tabagismo, de acordo com Mendes, Clara e Pádua (2004), é considerado o inimigo público número um do coração. De fato, o tabagismo é duplamente prejudicial, pois não só aumenta duas a quatro vezes mais o risco coronário e de morte súbita (Martinet \& Bohadana, 2001/2003), como também agrava outros fatores de risco cardiovascular (FRCV)

\footnotetext{
1 Este trabalho é derivado da Dissertação de Mestrado defendida pela primeira autora, sob a orientação da segunda, junto ao Mestrado Integrado em Psicologia pela Faculdade de Psicologia e de Ciências da Educação da Universidade do Porto. Esse texto foi revisado, seguindo o Acordo Ortográfico da Língua Portuguesa (1990), em vigor a partir de $1^{\circ}$ de janeiro de 2009

2 Endereço para correspondência:

Vânia Rocha. Rua Conde Alto Mearim 829. $1^{\circ}$ D. 4450-035 Matosinhos,
} Portugal.E-mail: vaniarocha.psi@gmail.com que contribuem para o desenvolvimento das doenças coronarianas, nomeadamente a hipertensão (HTA), dislipidemia e Diabetes mellitus (Mendes e cols., 2004). Estas informações tornam-se mais relevantes quando se toma conhecimento de que $30 \%$ da população portuguesa é fumante e $20 \%$ dos infartos ocorrem em fumantes (Mendes e cols., 2004). FerreiraBorges e Filho (2004) acrescentam a estimativa de que 17 a $30 \%$ da mortalidade cardiovascular esteja diretamente relacionada com a dependência do tabaco. No entanto, ressalta-se que deixar de fumar imediatamente após um infarto, diminui em $40 \%$ o risco de morte ao fim de um ano (Martinet \& Bohadana, 2001/2003), de tal forma que, 10 a 15 anos após um infarto, o risco de mortalidade cardiovascular dos antigos fumantes é comparável aos que nunca fumaram (Mendes e cols., 2004). Deste modo, Trigo e Rocha (2002) afirmam que a história de 
infarto constitui um momento, no qual o doente se revela particularmente receptivo à interrupção do hábito de fumar. De fato, após a vivência pessoal e profunda em que consiste o infarto e a sensação de ameaça de vida a ele associado, o doente começa a valorizar o papel que o tabagismo desempenha na sua doença (Perez, Nicolau, Romano, \& Laranjeira, 2007), passando a tornar-se convicto da sua própria vulnerabilidade (Trigo \& Rocha, 2002). Assim, ao estarem conscientes da severidade da sua doença, estão também motivados a melhorar o seu prognóstico (van Berkel, van der Vlugt, \& Boersma, 2000). Deste modo, é essencial que os profissionais da área de saúde mental em consultas de cardiologia estejam, não só sensibilizados para esta questão, como também bem informados acerca dos mecanismos físicos e psíquicos desta adição, nomeadamente da existência da dependência fisiológica e comportamental do tabagismo.

\section{A dependência fisiológica e a dependência comportamental do tabagismo}

A nicotina é a responsável pela dependência fisiológica do tabagismo, visto esta ser sustentada pelos efeitos positivos que advêm da passagem rápida desta substância para o cérebro e que reforçam o comportamento de ingestão da droga (Martinet \& Bohadana, 2001/2003). A nicotina entra na corrente sanguínea através dos pulmões e demora, em média, dez segundos para atingir o Sistema Nervoso Central (SNC) através dos receptores nicotínicos (FerreiraBorges \& Filho, 2004). A ativação do SNC leva ao aumento de dopamina no sistema mesolímbico de recompensas (implicado também no estabelecimento de dependência a outras drogas), resultando numa sensação de prazer e bemestar, o que se torna um reforço para que o fumante, após algum tempo, volte a fumar (Gomes, 2007). Desta forma, a falta de nicotina produz reações desagradáveis no organismo que se denominam de "síndrome de abstinência", sendo caracterizada por dores de cabeça, tremores, sudorese, ansiedade elevada, irritabilidade, dificuldade de concentração e até insônias (Ferreira-Borges \& Filho, 2004). Assim, a dependência fisiológica significa a perda de controle sobre o uso de determinada droga, visto que o fumante não consegue exercer autocontrole relativamente ao uso do cigarro, ainda que esteja totalmente consciente dos sérios riscos que esse hábito acarreta na sua saúde (Ferreira-Borges \& Filho, 2004).

No entanto, a dependência tabágica é algo mais do que os seus efeitos fisiológicos mensuráveis. Com efeito, a variabilidade entre fumantes e a sua evolução tabágica explicam-se por fatores sociais e psicológicos, que influenciam a percepção dos efeitos físicos, o que aponta para a existência de uma dependência comportamental (Slama, 2001/2003). Segundo Ferreira-Borges e Filho (2004), a dependência comportamental resulta da rotina que o fumante estabelece, progressivamente, no seu consumo de tabaco, associando o hábito de fumar a algumas atividades, como por exemplo, fumar e tomar café, fumar após as refeições ou fumar antes de dormir. De fato, a dependência comportamental traduz a associação entre os reforços subjetivos da nicotina e certas situações, emoções ou contextos, que se tornam dissociados dos reforços físicos (Slama, 2001/2003). Assim, o tabagismo conduz não só às adaptações neurológicas provocadas pela nicotina, mas também a interpretações cognitivas e a associações afetivas no contexto dos antecedentes ou consequências do ato de fumar (Slama, 2001/2003). Desta forma, pode concluir-se que as cognições e as emoções têm um papel muito importante no comportamento tabagista, deixando transparecer a influência que certas variáveis psicossociais podem exercer neste âmbito, encontrando-se, entre estas, a assertividade e a alexitimia.

\section{Assertividade}

O comportamento assertivo corresponde à capacidade do indivíduo em afirmar-se nas interações sociais, defender os seus direitos, expressar as suas opiniões, sentimentos, necessidades e insatisfações e solicitar mudanças de comportamento das outras pessoas, sem desrespeitar os direitos das mesmas (Bandeira \& Ireno, 2002). De fato, os mesmos autores referem que o comportamento assertivo é fundamental em diversas interações sociais que ocorrem nas situações quotidianas e contribui para o desenvolvimento das relações interpessoais, melhorando o funcionamento social e a qualidade de vida das pessoas.

A falta de assertividade tem vindo a ser relacionada com o padrão comportamental tipo $\mathrm{A}$, descrito como um fator de risco para a ocorrência das doenças coronarianas (Fontaine, Kubertus, \& Étienne, 1996/1998). Fontaine e cols. (1996/1998) explicitam que o indivíduo com padrão comportamental tipo A é alguém que procura o estresse, cujo estilo de vida o coloca em situações estressoras, reforçando a sua hiperatividade autónoma, e levando, consequentemente, a um aumento do risco cardiovascular. Para Megargee (conforme citado por Fontaine e cols., 1996/1998), a hostilidade pode tornar-se um meio de comunicação para o doente cardíaco que apresente este padrão comportamental, sobretudo quando se sente frustrado perante um objetivo não atingido, demonstrando, assim, um déficit de assertividade na comunicação.

Porém, Denollet (1997) identificou a personalidade tipo $\mathrm{D}$ que se caracteriza por altos níveis de ansiedade, tensão crônica, emoções negativas e inibição social, aumentando o estresse emocional e levando a um baixo nível de bem-estar psicológico. No estudo desenvolvido por Denollet (2005), a personalidade tipo $\mathrm{D}$ foi mais prevalente entre pacientes cardíacos e hipertensos comparativamente com indivíduos da população geral. Denollet (1997) afirma que não só a hostilidade, como também a depressão e a ansiedade, podem estar associadas à incidência de doenças crônicas, incluindo doenças cardiovasculares. Atualmente, assume-se que os sintomas da depressão estão associados a um aumento do risco a longo-prazo da mortalidade cardíaca e da possibilidade de 
novo infarto. Segundo o autor, os indivíduos socialmente inibidos apresentam não só uma auto-expressão reduzida, como também tendem a diminuir a disponibilidade do suporte social e sentem-se inseguros com os outros, experienciando falta de assertividade.

Quanto à relação da assertividade com o consumo de substâncias, Biglan, Weissman e Severson (1985) indicam que o tabagismo pode ser prevenido por meio de técnicas que promovam a assertividade, nomeadamente aquelas que são relevantes para lidar com a pressão social e com as situações de conflito interpessoal. Além disso, Van Hasselt, Null, Kempton e Bukstein (conforme citado por López-Torrecillas, García, Garcia, Izquierdo, \& Sánchez-Barreira, 2000) demonstraram que indivíduos que apresentam dificuldades em interagir socialmente e em lidar com situações de risco, podem tornar-se dependentes de drogas.

\section{Alexitimia}

O termo alexitimia foi introduzido por Sifneos em 1972 e significa, literalmente, sem palavras para as emoções (Souto, 2000). Nemiah (conforme citado por Amorim, Guerra, \& Maciel, 2005) considerou como características mais relevantes nos sujeitos alexitímicos: a dificuldade em identificar e descrever emoções e afetos; a pobreza imaginativa e dificuldade em fantasiar; o pensamento concreto orientado para o exterior; e o estilo de vida mais voltado para a ação. É importante salientar que as consequências dos déficits emocionais da alexitimia se estendem além das dificuldades intrapessoais (Zackheim, 2007). Com efeito, a alexitimia dificulta o estabelecimento de relações interpessoais, visto os alexitímicos exibirem falta de empatia ou déficit em compreender e experienciar as emoções dos outros (Lumley, Ovies, Stettner, Wehmer, \& Lakey, 1996). De acordo com Dunn e Brown (conforme citado por Souto, 2000), os indivíduos alexitímicos comunicam verbalmente o seu mal-estar emocional aos outros de forma muito empobrecida, falhando, pois, em considerar os outros como fontes de ajuda ou conforto. Kojima, Frasure-Smith e Lespérance (2001), assim como Lumley, Ovies e cols. (1996) concluíram que os alexitímicos consideravam ter um suporte social mais reduzido que os não alexitímicos, possuindo, efetivamente, uma rede social de apoio menor (poucas relações íntimas, como amigos, namorados, ou um companheiro estável). Assim, devido às suas competências sociais reduzidas, os alexitímicos demonstram dificuldade em formar e manter relações próximas (Lumley, Ovies e cols., 1996), que ajudam a diminuir o estresse (Kojima e cols., 2001), exacerbando, deste modo, a vulnerabilidade para as doenças somáticas (Zackheim, 2007).

No estudo de Amorim e cols. (2005) e Kojima e cols. (2001), a alexitimia foi mais observada em pacientes que sofreram infarto do que na população saudável, o que aponta para a existência de uma relação entre alexitimia e este evento. $\mathrm{Na}$ amostra constituída por doentes cardíacos do estudo de Valkamo, Hintikka, Honkalampi, Niskanen, Koivumaa-Honkanen, \& Viinamäki (2001), 21\% dos sujeitos eram alexitímicos. Contudo, a alexitimia pode também ser percebida como consequência do próprio infarto, pois há uma grande probabilidade da capacidade de modulação de emoções dos indivíduos que sofreram infarto sair afetada, devido à importância deste evento na adaptação dos sujeitos (Cardoso, 1984). Além disso, a depressão, a ansiedade e a hostilidade são significativamente mais elevadas nos alexitímicos do que nos não-alexitímicos (Kojima e cols., 2001.). Assim, poderá dizer-se que a alexitimia está associada à redução da satisfação com a vida em doentes com doenças coronarianas, contribuindo consequentemente para o fardo psicossocial associado à doença crônica (Valkamo e cols., 2001).

Lumley, Stettner e Wehmer (1996) afirmam que a alexitimia pode levar indiretamente à doença orgânica, através de comportamentos pouco saudáveis como o tabagismo, nutrição pobre, um estilo de vida sedentário, uma má adesão ao regime terapêutico e abuso de substâncias. Com efeito, estes autores colocaram a hipótese de que, devido às limitações cognitivas em identificar e elaborar emoções, os indivíduos alexitímicos possuem sensações não controláveis que podem resultar em comportamentos aditivos para auto-regular as emoções disruptivas, e compensar tanto a incapacidade de modelar afetos perturbadores (Finn, Martin, \& Phil conforme citado por Speranza e cols., 2004), como os déficits na defesa de afetos (Souto, 2000). A dependência por si mesma reduz a capacidade dos alexitímicos em decodificar emoções, limitando o sujeito, cada vez mais, a um funcionamento rígido (Speranza e cols., 2004).

A associação da alexitimia com afetos negativos é de particular importância nas perturbações de abuso de substâncias, sendo particularmente problemática a associação entre a alexitimia e a hostilidade (Donker, Bretele, \& van der Staak, 2000). Efetivamente, a confirmação da associação entre a alexitimia e a hostilidade suporta o ponto de vista de que as pessoas que abusam de substâncias apresentam um duplo problema paradoxal com emoções - a incapacidade em identificar e expressar sentimentos e a vasta experiência de afetos negativos severos - o que suporta a utilização de técnicas de gestão de raiva no tratamento do consumo de substâncias (Marlatt, 1996).

Existe também evidência de que a assertividade e a alexitimia estejam relacionadas. Lipp, Frare e Santos (2007) revelam que a alexitimia, a falta de assertividade e a tendência à agressividade têm sido identificadas clinicamente em muitos pacientes hipertensos, sendo fatores importantes na determinação da reatividade cardiovascular que ocorre em contatos sociais estressantes.

\section{Variáveis e Objetivos}

Apesar das inequívocas vantagens associadas à interrupção do consumo de tabaco, a maior parte dos programas 
elaborados com este propósito revela-se ineficaz e com elevadas taxas de recaída (Trigo \& Rocha, 2002). Uma possível explicação está ligada ao fato de não existir muita investigação sobre o tipo preponderante de dependência tabágica nos doentes que sofreram infarto, assim como ainda ser insuficientemente explorada a sua relação com a alexitimia e a assertividade e a relação entre ambas. No presente estudo, foram selecionadas como variáveis ambas as dependências fisiológica e comportamental da nicotina, a assertividade e a alexitimia. Como objetivos do estudo foram definidos os seguintes: (a) caracterizar a dependência fisiológica e a dependência comportamental da nicotina, bem como a assertividade e a alexitimia numa amostra diagnosticada com infarto do miocárdio, (b) analisar a relação entre a dependência fisiológica e a dependência comportamental da nicotina, bem como a assertividade e a alexitimia numa amostra diagnosticada com infarto do miocárdio.

\section{Método}

\section{Participantes}

A amostra é propositadamente constituída por 30 sujeitos do sexo masculino, não só por razões metodológicas de controle de variáveis, como também pelo fato do tabagismo e das doenças coronarianas serem mais frequentes no sexo masculino (Amorim e cols., 2005). A faixa etária (dos 40 aos 67 anos) foi também controlada previamente para englobar o intervalo etário considerado como a "meia-idade" (Amorim e cols., 2005) e por ser elucidativo do tipo de população característica do serviço institucional público onde os dados foram recolhidos $(M=52,40 ; D P=7,72)$. Optou-se também por incluir apenas os sujeitos internados no Serviço de Cardiologia do Hospital de São João que apresentavam o diagnóstico de infarto do miocárdio nos seus processos clínicos, não só pela existência de estudos que verificaram que os pacientes com infarto apresentavam uma taxa mais elevada de tabagismo comparativamente com aqueles com diagnóstico de angina instável, mas também devido à confirmação da presença de alexitimia e falta de assertividade nos sujeitos que sofreram este evento (Guerra, Viana, Fernandes, \& Maciel, 2007; Valkamo e cols., 2001). Relativamente ao nível de escolaridade, a média situa-se aproximadamente no $6^{\circ}$ ano de escolaridade $(M=6,43 ; D P=3,55)$, com variação de sujeitos sem qualquer escolaridade até indivíduos com o $12^{\circ}$ ano. Vale salientar que o fato da amostra ser constituída por 30 sujeitos e ser de conveniência (limitada a uma unidade de cuidados do Hospital de São João), ou seja, não ter sido selecionada aleatoriamente, impede que se extrapolem os dados para a população em geral.

\section{Material}

Quanto aos materiais utilizados, foram aplicados quatro instrumentos de auto-preenchimento, de curta duração e fácil compreensão.
O Teste de Fagerström para a Dependência Nicotínica, denominado originalmente por Fagerström Test for Nicotine Dependence (FTND) é um teste composto por seis itens, de administração fácil e de grande utilidade para conhecer o nível de dependência nicotínica do indivíduo, apresentando níveis aceitáveis de consistência interna (Heatherton, Kozlowski, Frecker, \& Fagerström, 1991). A pontuação varia entre 0 e 10: um valor compreendido entre 0 e 2 indica uma dependência fisiológica reduzida, entre 3 a 6 corresponde a uma dependência moderada, enquanto que de 7 a 10 traduz uma dependência fisiológica elevada.

O Questionário Glover-Nilsson para a Dependência Comportamental, ou Glover-Nilsson Smoking Behavioral Questionnaire (GNSBQ), é constituído por 11 itens e pretende, não só identificar o nível de dependência comportamental de um fumante, mas também estimar em que grau será mais adequada uma intervenção comportamental na interrupção do tabagismo (Glover, Nilsson, Westin, Glover, Laflin, \& Persson, 2005). A pontuação varia de 0 a 44, sendo definidos vários grupos, a saber: $<12$ dependência comportamental reduzida; 12-22 dependência comportamental moderada; 23-33 dependência comportamental elevada; $>33$ dependência comportamental muito elevada (Glover e cols., 2005). Embora o GNSBQ e o FTND sejam utilizados correntemente na prática clínica, não existe ainda em Portugal uma adaptação psicométrica publicada para estes dois instrumentos.

A Escala de Assertividade de Rathus, na sua denominação original Rathus Assertiveness Schedule (RAS), tem como objetivo principal medir o comportamento assertivo com base numa avaliação de 30 afirmações (Detry \& Castro, 1996), tendo o indivíduo que assinalar a sua resposta numa escala de Likert de seis pontos (de 0 a 5) que vai de "Nada característica" até "Muito característica". Relativamente à cotação, cada resposta do participante corresponde a um determinado valor na escala de cotação que vai de 3 a -3 . Deste modo, o ponto 5 da escala de Likert equivale a uma cotação de 3 e o ponto 0 corresponde a uma cotação de -3. Os resultados obtidos na investigação de Rathus (conforme citado por Detry \& Castro, 1996) e na validação da escala para a amostra portuguesa são semelhantes, correspondendo a média da população portuguesa a -1.83 e o desvio-padrão a 23.67 (Detry \& Castro, 1996). Assim, os resultados superiores a -1.83 indicam falta de assertividade, ao passo que valores abaixo desta média revelam pessoas assertivas.

A Escala de Alexitimia de Toronto de 20 itens, ou Toronto Alexithymia Scale - 20 items (TAS-20), é a escala com melhor validade e precisão no estudo dos déficits cognitivos e afetivos da alexitimia (Torres, 2005). Esta escala é composta por 20 itens, sendo as respostas assinaladas numa escala tipo Likert de cinco pontos (de 1 a 5). Quanto aos resultados, estes variam de 20 a 80 , sendo considerados alexitímicos os indivíduos com resultados superiores ou iguais a 61 e não 
alexitímicos os sujeitos com resultados inferiores ou iguais a 51 (Taylor e cols. conforme citado por Torres, 2005). Os resultados compreendidos entre 52 e 60, inclusive, correspondem a uma zona fronteira. A versão portuguesa utilizada neste estudo é a de Prazeres, Parker e Taylor (conforme citado por Torres, 2005) que possui uma adequada consistência interna $(0,798)$. A equivalência da tradução face ao original foi garantida por um resultado total de $\mathrm{r}=.91(\mathrm{p}<.001)$ (Amorim e cols., 2005), sustentando a utilização da TAS-20 em populações portuguesas (Torres, 2005).

\section{Procedimento de coleta de dados}

A Comissão de Ética para a Saúde do Hospital de S. João aprovou por unanimidade a presente investigação no dia 21 de Janeiro de 2008.

A aplicação dos instrumentos foi realizada com cada um dos participantes internados no Serviço de Cardiologia do referido hospital, que contemplavam os critérios adotados para a composição da amostra.

Vale salientar que, inicialmente, foi dada uma breve explicação aos indivíduos sobre a investigação em causa, tendo sido assegurada a sua confidencialidade e obtido o consentimento informado de todos os participantes, de acordo com a Declaração de Helsinque. Foi também referida a inexistência de respostas certas ou erradas. Pediu-se aos participantes que respondessem a todas as questões de uma forma honesta, informando-os que não havia restrição de tempo para o seu preenchimento e que, em caso de dúvidas, podiam solicitar esclarecimentos à investigadora.

\section{Procedimento de análise dos dados}

Para análise estatística dos dados foi utilizado o programa SPSS (Statistical Package for Social Sciences), versão 15.0. Para tal, foram realizadas correlações $r$ de Pearson, de modo a avaliar as associações entre as variáveis, e o teste $t$ de Student, para comparação entre grupos. O nível de significância adotado foi de $\mathrm{p}<0,05$.

\section{Resultados}

A Tabela 1 apresenta a caracterização da dependência fisiológica, da dependência comportamental, da assertividade e da alexitimia na amostra. Ao analisar a distribuição dos resultados obtidos através da aplicação do FTND, de acordo com os valores de corte do instrumento, verifica-se que a amostra é constituída principalmente por sujeitos com dependência fisiológica moderada $(n=17)$. Nos resultados referentes ao instrumento GNSBQ, observa-se que há mais sujeitos que apresentam uma dependência comportamental reduzida $(\mathrm{n}=14)$, seguindo-se de 12 sujeitos com dependência comportamental moderada. Quanto aos resultados obtidos através da RAS de acordo com o valor de corte do instrumento, a Tabela 1 aponta a falta de assertividade como uma característica predominante da amostra $(n=22)$. Relativamente aos resultados da TAS-20, é curioso observar que a amostra se distribui igualitariamente pelas três categorias da escala propostas, tendo em vista o valor de corte.

Tabela 1

Resultados do FTND, GNBSQ, RAS e TAS-20

\begin{tabular}{lccc}
\hline & $\mathbf{n}$ & $\mathbf{\%}$ & $\begin{array}{c}\% \\
\text { Acumulada }\end{array}$ \\
\hline Dependência fisiológica reduzida & 4 & 13,3 & 13,3 \\
Dependência fisiológica moderada & 17 & 56,7 & 70,0 \\
Dependência fisiológica elevada & 9 & 30,0 & 100,0 \\
$\quad$ Total & 30 & 100,0 & \\
Dependência comportamental reduzida & 14 & 46,7 & 46,7 \\
Dependência comportamental & 12 & 40,0 & 86,7 \\
moderada & 4 & 13,3 & 100,0 \\
Dependência comportamental elevada & 30 & 100,0 & \\
$\quad$ Total & 8 & 26,7 & 26,7 \\
Assertivo & 22 & 73,3 & 100,0 \\
Não assertivo & 30 & 100,0 & \\
$\quad$ Total & 10 & 33,3 & 33,3 \\
Alexitímicos & 10 & 33,3 & 66,7 \\
Não alexitímico & 10 & 33,3 & 100,0 \\
Zona Fronteira & 30 & 100,0 & \\
Total & &
\end{tabular}

FTND: Teste de Fagerström para a Dependência Nicotínica; GNSBQ: Questionário de Glover-Nilsson para a Dependência Comportamental; RAS: Escala de Assertivida

As análises correlacionais deste estudo referem-se às associações entre os resultados dos diferentes instrumentos. Observou-se, através da Tabela 2, que os resultados do FTND estão correlacionados positivamente com os resultados obtidos a partir do GNSBQ. Assim, quanto maior for a dependência fisiológica de nicotina, maior é também a dependência comportamental. Além dos resultados do GNSBQ estarem correlacionados com os do FTND, foi encontrada uma correlação positiva com os resultados da TAS-20. Deste modo, a dependência comportamental está associada à alexitimia. Não foi encontrada uma relação significativa entre os resultados do GNSBQ e os da RAS. No que diz respeito à assertividade, saliente-se a correlação positiva com a alexitimia. Com efeito, os sujeitos menos assertivos são os mais alexitímicos. Esta variável não está significativamente relacionada com os resultados encontrados através da aplicação do FTND e do GNSBQ. 
Tabela 2

Correlações entre os resultados obtidos por meio dos instrumentos FTND, GNSBQ, RAS e TAS-20 (n=30)

\begin{tabular}{lcccc}
\hline & Resultado do FTND & Resultado do GNSBQ & Resultado da RAS & Resultado da TAS-20 \\
\hline Resultado do FTND & 1 &, $492(* *)$ &,- 164 &,- 135 \\
Resultado do GNSBQ &, $492(* *)$ & 1 &, 158 &, $412(*)$ \\
Resultado da RAS &,- 164 &, 158 & 1 &, $392(*)$ \\
Resultado da TAS-20 &,- 135 &, $412(*)$ &, $392(*)$ & 1 \\
\hline
\end{tabular}

FTND: Teste de Fagerström para a Dependência Nicotínica; GNSBQ: Questionário de Glover-Nilsson para a Dependência Comportamental; RAS: Escala de Assertividade de Rathus; TAS-20: Escala de Alexitimia de Toronto-20 itens

$* p<.05 ; * * p<.01$ (Coeficiente de Pearson)

Em função dos resultados encontrados previamente, decidiu-se agrupar na mesma categoria os sujeitos que apresentavam uma dependência comportamental moderada e elevada segundo os valores de corte do GNSBQ, de modo a observar se existiriam diferenças significativas entre estes e os sujeitos com uma dependência comportamental reduzida, relativamente aos resultados dos demais instrumentos (ver Tabela 3). Note-se que há diferenças significativas no que concerne à dependência fisiológica e à alexitimia, ou seja, os sujeitos com uma dependência comportamental moderada/elevada apresentaram uma média superior tanto em relação à dependência fisiológica, quanto à alexitimia. Relativamente à alexitimia, a diferença é mais notória, assumindo-se os sujeitos com uma dependência comportamental reduzida como "não alexitímicos", enquanto que a média dos sujeitos com uma dependência comportamental moderada/elevada se situa na zona fronteira. Não se encontraram diferenças significativas no que diz respeito às médias obtidas através da aplicação da RAS.

Tabela 3

Diferenças nos resultados obtidos por meio dos instrumentos FTND, RAS e TAS-20 em função da dependência comportamental reduzida e moderada/elevada

\begin{tabular}{lccccccc}
\hline & \multicolumn{2}{c}{$\begin{array}{c}\text { Dependência reduzida } \\
(\mathbf{n}=\mathbf{1 4})\end{array}$} & & \multicolumn{2}{c}{$\begin{array}{c}\text { Dependência moderada/ } \\
\text { elevada (n=16) }\end{array}$} & & \\
\cline { 2 - 3 } & $\mathrm{M}$ & $\mathrm{DP}$ & & $\mathrm{M}$ & $\mathrm{DP}$ & & \\
\hline Resultados do FTND & 3,93 & 1,90 & & 5,81 & 2,11 & $\mathrm{t}=2,558$ & $\mathrm{p}<.05$ \\
Resultados da RAS & 5,57 & 31,13 & & 17,31 & 29,20 & $\mathrm{t}=1,066$ & $\mathrm{p}=\mathrm{ns}$ \\
Resultados da TAS-20 & 48,43 & 12,76 & & 57,31 & 10,38 & $\mathrm{t}=2,103$ & $\mathrm{p}<.05$ \\
\hline
\end{tabular}

Nota: Dependência reduzida $(M=6,93 ; D P=3,08)$; Dependência moderada/elevada $(M=18,38 ; D P=5,40)$

FTND: Teste de Fagerström para a Dependência Nicotínica; RAS: Escala de Assertividade de Rathus;

TAS-20-Escala de Alexitimia de Toronto-20 itens

n.s. $(p>.05)(t$ de Student)

\section{Discussão}

Os objetivos deste estudo passam pela caracterização da amostra relativamente às quatro variáveis selecionadas e pela análise da relação que estabelecem entre si. Fraga e cols. (2005) remetem para o estudo do EpiPorto, realizado entre 1999 e 2000, no qual se avaliou o grau de dependência nicotínica, através do Teste de Fagerström, numa amostra alargada de fumantes da cidade do Porto. Foi encontrado que metade da amostra do estudo do EpiPorto (50\%) apresenta uma dependência fisiológica reduzida, enquanto $56,7 \%$ dos doentes com infarto manifestam uma dependência fisiológica moderada. A percentagem de sujeitos com uma dependência fisiológica elevada é também superior na amostra do presente estudo (30\%) comparativamente com os participantes do estudo do EpiPorto (22,7\%). Deste modo, pode-se dizer que os fumantes que sofreram infarto apresentam um grau mais elevado de dependência fisiológica quando comparados com os sujeitos saudáveis. No entanto, não se encontraram dados de amostras saudáveis da população portuguesa a quem tivesse sido aplicado o Questionário Glover-Nilsson, e que, deste modo, permitissem avaliar se a amostra com o diagnóstico de infarto apresenta também valores mais elevados de dependência comportamental. Destaca-se que foi confirmada a existência de uma relação positiva entre a dependência fisiológica e a comportamental.

Ainda em relação à caracterização da amostra no que concerne às variáveis em estudo, é fundamental referir que 
os resultados obtidos indicam que a maioria da amostra não é assertiva (73\%), tal como o observado em investigações anteriores, como a de Donker e cols. (2000) que chamaram a atenção para a hostilidade, característica do padrão comportamental tipo A, nos indivíduos com doenças coronarianas; assim como Denollet (1997) que alertou para a existência da personalidade tipo D caracterizada, entre outros, pela inibição social. Lipp e cols. (2007) verificaram que a falta de assertividade, definida por Wolpe (conforme citado por Lipp e cols., 2007) como a inabilidade, não só de defender os direitos pessoais e opiniões, mas também de expressar afetos assertivamente, é uma característica prevalente em muitos hipertensos.

No que concerne à alexitimia, pode dizer-se que os resultados são concordantes com a investigação que confirma a presença desta variável em populações que foram diagnosticadas com infarto. Neste contexto, 33\% da amostra (um terço) é alexitímica, sendo um resultado idêntico ao encontrado por Amorim e cols. (2005), percentagem semelhante ao do estudo de Kojima e cols. (2001) em que 31\% dos sujeitos com infarto eram alexitímicos, assim como no de Valkamo e cols. (2001), em que a prevalência de alexitímicos em pacientes com doenças coronarianas foi de $21 \%$.

Relativamente à correlação positiva encontrada entre a dependência fisiológica e a comportamental, Guerra e cols. (2007) sugerem que o consumo persistente de nicotina pode causar dependência fisiológica independentemente da natureza comportamental ou fisiológica dos fatores que contribuem para a sua manutenção. Esta associação foi também referida por autores como Perriot e Barthélémy (2001/2003), que classificam o tabagismo como um comportamento adquirido que combina uma dependência comportamental e fisiológica, na qual a nicotina ocupa o lugar central e cuja manutenção é reforçada por diversos estímulos internos e ambientais. Biglan e cols. (1985) sugerem que influências sociais e comportamentais são fatores importantes na manutenção do ato de fumar, durante o período em que a tolerância e a dependência se desenvolvem. Há, portanto, uma relação interdependente entre a dependência fisiológica e a comportamental, que se evidenciou no presente estudo. No entanto, não se encontrou uma correlação entre a assertividade e a dependência comportamental, o que contradiz o estudo de Shiffman (1985) que afirma que face a situações estressantes, as pessoas que não possuem competências assertivas, recorrem a comportamentos aditivos como tentativa de lidar com os afetos negativos. Porém, pode-se supor uma ligação indireta entre a assertividade e a dependência comportamental mediada pela alexitimia.

A alexitimia está correlacionada positivamente com a dependência comportamental e com a assertividade, isto é, quanto mais alexitímicos são os sujeitos, menos assertivos e mais dependentes comportamentais são, o que está concordante com os resultados de Fernandez Gomez, Espina Eizaguirre e Aresti (1997) que concluíram que a alexitimia se encontra correlacionada com falta de assertividade, variáveis da história de consumo e gravidade de dependência. Efetivamente, algumas investigações realizadas ajudam a compreender a relação estabelecida entre a alexitimia e a falta de assertividade na população cardíaca. Amorim e cols. (2005) destacam a associação entre o infarto e a inibição de emoções e comportamentos, como por exemplo, a interiorização da raiva (anger-in), que é frequentemente suporte da inexpressão que caracteriza a alexitimia. Denollet (2005) indica que esta inexpressão pode estar associada à inibição social característica da personalidade tipo D. No entanto, é também frequente os doentes com infarto apresentarem uma média superior quanto à exteriorização da raiva (anger-out) característica do padrão comportamental tipo A, isto é, comunicarem de forma agressiva (Donker e cols., 2000). Amorim e cols. (2005) sugerem como explicação para este fenomeno, a dificuldade do doente cardíaco em modelar as emoções de forma adequada, o que se correlaciona com a existência de alexitimia. Deste modo, conclui-se que tanto a inexpressão de afetos como a dificuldade em modelar as emoções características da alexitimia, se refletem num estilo de comunicação não assertivo.

Vale destacar que a relação encontrada entre a dependência comportamental e a alexitimia torna-se mais notória quando a dependência comportamental assume valores mais elevados. Embora se tenha observado que a dependência comportamental e a alexitimia estão correlacionadas positivamente, chegou-se também à conclusão que os sujeitos com uma dependência comportamental reduzida são "não alexitímicos". Portanto, pode dizer-se que esta relação é sustentada pelos valores mais elevados no GNSBQ.

De modo a clarificar as consequências desta conjuntura, Lumley, Stettner e Wehmer (1996) e Souto (2000) referem que para compensar as limitações cognitivas em auto-regular e modular as emoções perturbadoras e em defender afetos, os indivíduos alexitímicos recorrem a comportamentos aditivos. Diclemente e Prochaska (1985) referem que na iniciação e manutenção do hábito de fumar, os cigarros representam um mecanismo de enfrentamento (coping) paliativo que os indivíduos usam para gerir o estresse causado por outros problemas da sua vida. Assim, através de uma combinação de funções psicológicas e sociais, o ato de fumar reduz sentimentos de insegurança e de percepção de ameaça, aumenta o relaxamento e ajuda a controlar a ansiedade, o medo e a raiva (Leventhal \& Cleary conforme citado por Diclemente \& Prochaska, 1985). Efetivamente, para muitos, fumar torna-se uma "muleta" utilizada para regular respostas emocionais mobilizadas pelo estresse criado por exigências profissionais, familiares e sociais. Shiffman (1985) conclui igualmente que os fumantes são tentados a recorrer a cigarros como estratégia de enfrentamento (coping) para lidar com o estresse e com os afetos negativos, assim como para reduzir a tensão sentida. Tais fumantes atribuem o seu estresse emocional a fontes interpessoais e psicológicas, mas sentem que o ato de fumar os alivia. 
Se o tabagismo realmente reduz o afeto negativo é um assunto controverso (Shiffman, 1985). Uma série de experiências conduzidas por Marlatt (conforme citado por Shiffman, 1985) sugerem que as expectativas pessoais dos efeitos da droga podem ser mais importantes do que a própria farmacologia na determinação do valor funcional da mesma. Em suma, a utilização do tabaco como estratégia de enfrentamento (coping) para lidar com o estresse e emoções desagradáveis parece motivar frequentemente as recaídas e estimular o consumo de tabaco. Speranza e cols. (2004) indicam que, posteriormente, a dependência por si mesma vai reduzindo a habilidade dos alexitímicos em decodificar emoções, fechando o sujeito no seu funcionamento rígido.

\section{Considerações finais}

$\mathrm{Na}$ amostra constituída por doentes que sofreram infarto verificou-se que estes apresentam valores superiores de dependência fisiológica comparativamente às pessoas saudáveis (Fraga e cols., 2005). Constatou-se, também, a presença de alexitimia e falta de assertividade, pois a amostra demonstrou ser predominantemente não assertiva, além de um terço da mesma ser ainda alexitímica. Contudo, talvez o fato mais singular, que merece ser destacado, prende-se com a relação encontrada entre a dependência comportamental e a alexitimia, tornando-se esta relação mais expressiva à medida que a dependência comportamental assume valores mais elevados. Outro dado interessante diz respeito à relação positiva encontrada entre a assertividade e a alexitimia, que levanta a hipótese desta última desempenhar um efeito mediador entre a assertividade e a dependência comportamental. Assim, conclui-se que os sujeitos alexitímicos, que apresentam dificuldades na identificação e comunicação das suas emoções (Zackheim, 2007), não conseguem interagir socialmente de forma assertiva, o que está de acordo com certas características que compõem os padrões comportamentais tipo A e D frequentemente atribuídas aos doentes cardíacos, nomeadamente um potencial de raiva aumentado e inibição social (Denollet, 2005; Donker e cols., 2000;). Deste modo, e segundo Shiffman (1985), os indivíduos recorrem ao tabagismo, não só pelas suas propriedades químicas (dependência fisiológica), mas também pela expectativa de que o ato de fumar os aliviará do estresse e das emoções negativas presentes nas suas vidas (dependência comportamental).

Destaca-se algumas limitações do presente trabalho. Inicialmente, os controles efetuados relativamente ao sexo, idade e diagnóstico dos sujeitos restringem os resultados apenas aos indivíduos do sexo masculino, entre os 40 e os 67 anos, diagnosticados com infarto, e não a toda a população com patologia cardíaca. Além disso, não existe igualmente um grupo de controle, o que seria benéfico para validar se os resultados são, efetivamente, um reflexo das características comportamentais dos doentes cardíacos ou se os resultados seriam idênticos na população saudável.
É recomendável, pela gravidade do quadro clínico dos doentes que sofreram infarto, que estes abandonem definitivamente o consumo de tabaco (Mendes e cols., 2004), o que aponta para a necessidade de continuar a apostar em investigações que se centrem no efeito das variáveis psicossociais na dependência tabágica, mais concretamente em aprofundar o papel da alexitimia e da assertividade neste domínio e de outras variáveis com as quais estas poderão estar relacionadas, particularmente a raiva, o estresse, a depressão, a ansiedade e a inibição social (Guerra e cols., 2007). Ao compreender e explorar o que mantém o comportamento tabágico, assim como as causas que muitas vezes impossibilitam a manutenção da abstinência ao longo do tempo, abre-se caminho para a implementação de intervenções que incidam sobre áreas-problema apresentadas por estes sujeitos (Diclemente \& Prochaska, 1985). Assim, esta investigação confirma a necessidade de se privilegiar, na intervenção psicológica para a interrupção do hábito de fumar em doentes com infarto, o trabalho relativo às competências sociais e à expressão e regulação emocional. De fato, cabe ao psicólogo a função de trabalhar conjuntamente com o paciente todos estes aspectos (Amorim e cols., 2005), com a finalidade de possibilitar que este último aprenda a gerir situações e eventos estressantes de forma adequada, recorrendo, não ao tabagismo como mecanismo de enfrentamento (coping), mas sim a estratégias mais adaptadas e, sobretudo, promotoras da sua saúde física e mental.

\section{Referências}

Amorim, E., Guerra, M., \& Maciel, M. J. (2005). A alexitimia e a hostilidade em doentes que sofreram enfarte agudo do miocárdio. Revista Portuguesa de Psicossomática, 7 , 231-243.

Bandeira, M., \& Ireno, E. (2002). Reinserção social de psicóticos: Avaliação global do grau de assertividade em situações de fazer e receber crítica. Psicologia: Reflexão e Crítica, 15, 665-675.

Biglan, A., Weissman, W., \& Severson, H. (1985). Coping with social influences to smoke. In S. Shiffman \& T. A. Wills (Orgs.), Coping and substance use (pp. 95-116). Orlando: Academic Press.

Cardoso, R. M. (1984). Enfarte do miocárdio: Factores de risco da pessoa em situação. Tese de doutorado nãopublicada, Universidade do Porto, Porto.

Denollet, J. (1997). Personality, emotional distress and coronary heart disease. European Journal of Personality, $11,343-357$.

Denollet, J. (2005). DS14: Standard assessment of negative affectivity, social inhibition, and Type D personality. Psychosomatic Medicine, 67, 89-97.

Detry, B., \& Castro, S. L. (1996). A escala de assertividade de Rathus: Versão portuguesa. In L. Almeida, S. Araújo, M. Gonçalves, C. Machado, \& M. Simões (Orgs.), Avaliação Psicológica: Formas e Contextos (Vol. 4, pp. 357-363). Braga: Apport. 
Diclemente, C. C., \& Prochaska, J. O. (1985). Processes and stages of self-change: Coping and competence in smoking behavior change. In S. Shiffman \& T. A. Wills (Orgs.), Coping and substance use (pp. 319-343). Orlando: Academic Press.

Donker, F., Breteler, M., \& van der Staak, C. (2000). Assessment of hostility in patients with coronary heart disease. Journal of Personality Assessment, 75, 158-177.

Ferreira-Borges, C., \& Filho, H. C. (2004). Tabagismo: Manual técnico 3. Lisboa: Climepsi Editores.

Fontaine, O., Kubertus, H., \& Étienne, A. (1998). Stress e Cardiologia (P. Mendonça, Trad.). Lisboa: Climepsi Editores. (Original publicado em 1996)

Fraga, S., Sousa, S., Santos, A. C., Mello, M., Lunet, N., Padrão, P., \& Barros, H. (2005). Tabagismo em Portugal. Arquivos de Medicina, 19, 207-229.

Glover, E. D., Nilsson, F., Westin, A., Glover, P. N., Laflin, M., \& Persson, B. (2005). Developmental history of the Glover-Nilsson Smoking Behavioral Questionnaire. American Journal of Health Behavior, 29, 443-455.

Gomes, I. (2007). Dependência. In J. Agostinho Marques (Org.), Tabagismo (pp.77-83). Matosinhos: QuidNovi.

Fernandez Gomez, C., Espina Eizaguirre, A., \& Aresti, A. (1997). Alexitimia y características clínicas en abuso de opiáceos. Revista Toxicodependências, 3, 77-85.

Guerra, M. P., Viana, F., Fernandes, O., \& Maciel, M. J. (2007, Outubro). Assertiveness and motivation to quit smoking in cardiac patients after myocardial infarction. Trabalho apresentado na $9^{\text {th }}$ Annual Conference of SRNT. Madrid, Espanha.

Heatherton, T., Kozlowski, L., Frecker, R., \& Fagerström, K. O. (1991). The Fagerström Test for Nicotine Dependence: A revision of the Fagerström Tolerance Questionnaire. British Journal of Addiction, 86, 1119-1127.

Kojima, M., Frasure-Smith, N., \& Lespérance, F. (2001). Alexithymia following myocardial infarction Psychometric properties and correlates of the Toronto Alexithymia Scale. Journal of Psychosomatic Research, 51, 487-495.

Lipp, M., Frare, A., \& Santos, F. (2007). Efeitos de variáveis psicológicas na reactividade cardiovascular em momentos de stress emocional. Estudos de Psicologia, 24, 161-167.

López-Torrecillas, F., García, J., Garcia, M., Izquierdo, D., \& Sánchez-Barreira, M. (2000). Variables modulating stress and coping that discriminate drug consumers from low or nondrug consumers. Addictive Behaviors, 25, 161-165.

Lumley, M. A., Ovies, T., Stettner, L., Wehmer, F., \& Lakey, B. (1996). Alexithymia, social support and health problems. Journal of Psychossomatic Research, 41, 519-530.
Lumley, M. A., Stettner, L., \& Wehmer, F. (1996). How are alexithymia and physical illness linked? A Review and Critique of Pathways. Journal of Psychossomatic Research, 41, 505-518.

Marlatt, G. A. (1996). Taxonomy of high-risk situations for alcohol relapse: Evolution and development of a cognitive-behavioral model. Addiction, 91, 37- 49.

Martinet, Y., \& Bohadana, A. (2003). O Tabagismo: Da prevenção à abstinência (J. N. Almeida, Trad.). Lisboa: Climepsi Editores. (Original publicado em 2001)

Mendes, L., Clara, J. G., \& Pádua, F. (2004). Doenças cardiovaculares. In C. Ferreira-Borges \& H. C. Filho (Orgs.), Tabagismo: Manual técnico 3 (pp. 51-61). Lisboa: Climepsi Editores.

Perez, G., Nicolau, J., Romano, B., \& Laranjeira, R. (2007). Smoking-associated factors in myocardial infarction and unstable angina: Do gender differences exist? Addictive Behaviors, 32, 1295-1301.

Perriot, J., \& Barthélémy, L. (2003). Abordagens colectivas de auxílio à desabituação tabágica. In Y. Martinet \& A. Bohadana (Orgs.), O Tabagismo: Da prevenção à abstinência (J. N. Almeida, Trad., pp. 287-295). Lisboa: Climepsi Editores. (Original publicado em 2001)

Shiffman, S. (1985). Coping with temptations to smoke. In S. Shiffman \& T. A. Wills (Orgs.), Coping and Substance Use (pp. 223-242). Orlando: Academic Press.

Slama, K. (2003). Nicotina e dependência psicológica. In Y. Martinet \& A. Bohadana (Orgs.), O Tabagismo: Da prevenção à abstinência (pp. 99-107, J. N. Almeida, Trad.). Lisboa: Climepsi Editores. (Original publicado em 2001)

Souto, T. S. (2000). A alexitimia e a dependência de drogas. Os sentimentos, o discurso e as drogas. Dissertação de mestrado não-publicada, Universidade do Porto, Porto.

Speranza, M., Corcos, M., Stéphan, P., Loas, G., PérezDiaz, F., Lang, F., Venisse, J., Bizouard, P., Flament, M., Halfon, O., \& Jeammet, P. (2004). Alexithymia, depressive experiences, and dependency in addictive disorders. Substance Use \& Misuse, 39, 551-579.

Torres, S. (2005). O corpo e o silêncio das emoções. Estudo da alexitimia na anorexia nervosa. Tese de doutorado não-publicada, Universidade do Porto, Porto.

Trigo, M., \& Rocha, E. C. (2002). Cessação de hábitos tabágicos em doentes coronários. Acta Médica Portuguesa, 15, 337-344.

Valkamo, M., Hintikka, J., Honkalampi, K., Niskanen, L., Koivumaa-Honkanen, H., \& Viinamäki, H. (2001). Alexithymia in patients with coronary heart disease. Journal of Psychosomatic Research, 50, 125-130.

van Berkel, T., van der Vlugt, M., \& Boersma, H. (2000). Characteristics of smokers and long-term changes in smoking behavior in consecutive patients with myocardial infarction. Preventive Medecine, 31, 732-741. 
Zackheim, L. (2007). Alexithymia: The expanding realm of research. Journal of Psychosomatic Research, 63, 345-347.

Vânia Rocha é Mestre em Psicologia pelo Programa de Pósgraduação da Faculdade de Psicologia e de Ciências da Educação da Universidade do Porto, Portugal.

Marina Prista Guerra é Professora Associada da Faculdade de Psicologia e de Ciências da Educação da Universidade do Porto, Portugal.

Maria Júlia Maciel é Professora Associada da Faculdade de Medicina da Universidade do Porto, Portugal.

Recebido: 28/08/2009

$1^{a}$ Revisão: $23 / 11 / 2009$

$2^{a}$ Revisão: $13 / 02 / 2010$

Aceite final: $11 / 03 / 2010$ 